\title{
Assessment of climate change adaptation capacity of agriculture in Ho Chi Minh City
}

\author{
Linh T. Vu ${ }^{1 *}$, Ngoc T. A. Pham ${ }^{2}$, Dung M. Ho ${ }^{3}$, \& Loi K. Nguyen ${ }^{4}$ \\ ${ }^{1}$ Department of Natural Resources and Environment of Ho Chi Minh City, Vietnam \\ ${ }^{2}$ Faculty of Economics, Nong Lam University, Ho Chi Minh City, Vietnam \\ ${ }^{3}$ Institute for Environment and Resources, Vietnam National University, Ho Chi Minh City, Vietnam \\ ${ }^{4}$ Research Center for Climate Change, Nong Lam University, Ho Chi Minh City, Vietnam
}

ARTICLE INFO
Research Paper
Received: August 06, 2019
Revised: January 02, 2020
Accepted: April 24, 2020
Keywords
Adaptation capacity
Climate change
Ho Chi Minh City
Perception
Vulnerability
${ }^{*}$ Corresponding author
Vu Thuy Linh
Email: vtlinh.uk@gmail.com

\begin{abstract}
This research used sociological survey method combined with Likert scale and the factor analysis method to assess adaptability to climate change from 4 factors including awareness of climate change, experience in responding to climate change, government support and access to resources. Based on the results of social survey of 10 districts related to agricultural activities in Ho Chi Minh City, the research calculated the score for 4 criteria and zoning for climate change adaptation value for each district. The results showed that high and average -resilient districts were $\mathrm{Cu}$ Chi, 9 and 12. Cu Chi was rated to have the highest adaptability, with an overall adaptability value of 0.86 . Indeed, $\mathrm{Cu}$ Chi district was noted for having support from the government and access to the highest level of support among the 10 districts. Meanwhile, Binh Chanh, Go Vap and Thu Duc districts were located in a low adaptability area.
\end{abstract}

Cited as: : Vu, L. T., Pham, N. T. A., Nguyen, L. K., \& Ho, D. M. (2020). Assessment of climate change adaptation capacity of agriculture in Ho Chi Minh City. The Journal of Agriculture and Development 19(2), 79-89. 


\title{
Đánh giá khả năng thích ứng với biến đổi khí hậu cho nông nghiệp ở thành phố Hồ Chí Minh
}

\author{
Vũ Thùy Linh ${ }^{1 *}$, Phạm Thị Ánh $\mathrm{Ngọc}^{2}$, Hồ Minh Dũng ${ }^{3}$, \& Nguyễn Kim Lợi ${ }^{4}$ \\ ${ }^{1}$ Sở Tài Nguyên và Môi Trường, TP. Hồ Chí Minh \\ ${ }^{2}$ Khoa Kinh Tế, Trường Đại Học Nông Lâm TP.HCM, TP. Hồ Chí Minh \\ ${ }^{3}$ Viện Môi Trường và Tài Nguyên - ĐHQG TP.HCM, TP. Hồ Chí Minh \\ ${ }^{4}$ Trung Tâm Nghiên Cứu Biến Đổi Khí Hậu, Trường Đại Học Nông Lâm TP.HCM, TP. Hồ Chí Minh
}

\author{
THÔNG TIN BÀI BÁO \\ Bài báo khoa học \\ Ngày nhận: 06/08/2019 \\ Ngày chỉnh sửa: 02/01/2020 \\ Ngày chấp nhận: 24/04/2020 \\ Từ khóa \\ Biến đổi khí hậu \\ Khả năng thích ứng \\ Nhận thức người dân \\ Tính dễ bị tổn thương \\ Thành phố Hồ Chí Minh \\ *Tác giả liên hệ \\ Vũ Thùy Linh \\ Email: vtlinh.uk@gmail.com
}

\section{TÓM TẮT}

Trong bối cảnh nghiên cứu về các tác động của biến đổi khí hậu (BĐKH) nói chung và tác động đến nông nghiệp trên thế giới nói riêng ngày càng rõ nét, TPHCM đã có nhiều minh chứng về tác động của các hiện tượng thời tiết bất thường gây bất lợi rất lớn cho ngành nông nghiệp. Nghiên cứu sử dụng phương pháp điều tra xã hội học kết hợp với thang đo cảm nhận Likert, phương pháp phân tích nhân tố nhằm đánh giá khả năng thích ứng với $\mathrm{BDKH}$ từ 4 nhân tố lần lượt là nhận thức về $\mathrm{BDKH}$, kinh nghiệm ứng phó với $\mathrm{BDKH}$, sự hỗ trợ của chính quyền và khả năng tiếp cận các nguồn hỗ trợ. Thông qua điểm số tính toán từ kết quả điều tra cộng đồng 10 quận/huyện có hoạt động nông nghiệp của Thành phố Hồ Chí Minh và các cơ quan quản lý giúp nghiên cứu xây dựng điểm số tổng hợp cho 4 tiêu chí và phân vùng không gian cho giá trị thích ứng $\mathrm{BDKH}$ cho từng quận/huyện. Kết quả cho thấy, các quận huyện có khả năng thích ứng trung bình, cao tập trung ở các huyện Củ Chi, quận 9 và 12 . Củ Chi được đánh giá có khả năng thích ứng cao nhất, với giá trị khả năng thích ứng tổng thể là 0,86 . Quả thật, huyện Củ Chi được ghi nhận đồng thời có sự hỗ trợ từ chính quyền và khả năng tiếp cận các nguồn hỗ trợ cao nhất trong số 10 quận/huyện. Trong khi đó, Bình Chánh, Gò Vấp, Thủ Đức nằm trong khu vực có khả năng thích ứng thấp.

\section{1. Đặt Vấn Đề}

Thành phố Hồ Chí Minh được xem là một trong những đô thị lớn trên thế giới, với số dân tính đến năm 2015 đã đạt 8,1 triệu người và được dự báo sẽ tiếp tục gia tăng đến gần 9,5 triệu người trước năm 2050 (GSO, 2016). Tuy nhiên, trong bối cảnh biến đồi khí hậu $(\mathrm{BĐKH)}$ toàn cầu, TP.HCM đang phải đối mặt với nhiều thách thức lớn. Theo Ngân hàn phát triển Châu Á (ADB, 2010, 2013), BĐKH đang mang đến nhiều rủi ro cho TP.HCM. Theo đó, TP.HCM được xếp trong 10 thành phố hàng đầu trên thế giới có số dân bị ảnh hưởng nghiêm trọng bởi các tác động bất lợi từ sự thay dổi khí hậu.

Biến đổi khí hậu toàn cầu đã và đang ảnh hưởng trực tiếp cũng như gián tiếp đến nhiều khía cạnh khác nhau trong cuộc sống, các tác động này có thể là hữu hình hay vô hình. Bộ Tài nguyên và Môi trường đã công bố kịch bản $\mathrm{BĐKH}$ và nước biển dâng cho Việt Nam phiên bản 2016; trong đó, nếu mực nước biển dâng $1 \mathrm{~m}$ và không có các giải pháp ứng phó phù hợp, khoảng 17,8\% diện tích TP.HCM có nguy cơ bị ngập (MONRE, 2016). Theo báo cáo của Ban chỉ đạo thực hiện Kế hoạch hành động ứng phó $\mathrm{BĐKH,} \mathrm{trong} 6$ năm qua (2005 - 2010), trên địa bàn TP.HCM, BĐKH đã gây thiệt hại đối với sản xuất nông nghiệp, nhất là áp thấp nhiệt đới, bão, triều cường, mưa lớn gây ngập úng kéo dài (DONRE, 2010). Rõ ràng, sự biến đổi của nguồn nước, sự biến động và dị thường của thời tiết và khí hậu do $\mathrm{BĐKH}$ gây ảnh hưởng lớn đến nông nghiệp thành phố trong bối cảnh sản xuất nông nghiệp hiện nay của Việt Nam nói chung và TP.HCM nói riêng còn phụ thuộc rất nhiều vào thời tiết. 
Vì vậy, đánh giá tính tổn thương do $\mathrm{BĐKH} \mathrm{cho}$ nông nghiệp thành phố cần được tiến hành một cách hệ thống. Theo đó, đánh giá tính dễ bị tổ thương (TDBTT) do BĐKH dựa vào chỉ thị được xem là một phương pháp luận có nhiều ưu điểm. Cụ thể, theo Balica \& ctv. (2012), các chỉ thị có thể được sử dụng để xây dựng nhiều kịch bản khác nhau tùy theo từng đối tượng bị tác động. Hơn nữa, việc sử dụng các chỉ thị còn có thể nhận dạng và định lượng các đặc điểm vốn có của hệ thống bị phơi nhiễm với các hiện tượng khí hậu cực đoan.

Ngoài ra, theo Can \& ctv. (2013), việc sử dụng các chỉ thị còn có thể xác định được mức độ tổn thương riêng cho từng vùng cụ thể, tạo điều kiện để so sánh về mặt không gian trong đánh giá tổn thương. Nhìn chung, các chỉ thị có thể xem như từng mảnh ghép, do đó khi sử dụng phương pháp luận dựa vào chỉ thị sẽ cung cấp một bức tranh tổng thể về tính tổn thương do $\mathrm{BĐKH}$ cho một đối tượng nhất định. Vì vậy, phương pháp luận này đã và đang được áp dụng thành công trong nhiều nghiên cứu đánh giá tính tổn thương trong và ngoài nước.

Ngoài ra, mặc dù TDBTT đã được định nghĩa theo nhiều quan điểm khác nhau và cho đến nay vẫn chưa có một khái niệm nào được xem là chuẩn nhất vì mức độ phù hợp phụ thuộc vào mục tiêu cụ thể trong từng trường hợp nhất định. Vì vậy, nghiên cứu này kế thừa khái niệm tính tổn thương của IPCC: "là mức độ một hệ thống bị nhạy cảm hoặc không thể chống chịu trước các tác động có hại của $\mathrm{BĐKH,} \mathrm{bao} \mathrm{gồm} \mathrm{dao} \mathrm{động} \mathrm{khí} \mathrm{hậu} \mathrm{và}$ các hiện tượng khí hậu cực đoan" (IPCC, 2014). Theo định nghĩa mới nhất này, TDBTT sẽ bao gồm tham số phợi nhiễm (Exposure $-\mathrm{E}$ ), tính nhạy cảm (Sensitivity - S) và khả năng thích ứng (Adaptive Capacity - AC). Trong đó, yếu tố khả năng thích ứng là mức độ mà hệ thống có thể làm giảm thiệt hại do tác động tiêu cực của $\mathrm{BĐKH}$ hoặc tận dụng các cơ hội do các tác động tích cực mang lại và khi các biện pháp thích ứng được tăng cường thì TDBTT theo đó sẽ giảm đi (Hình 1).

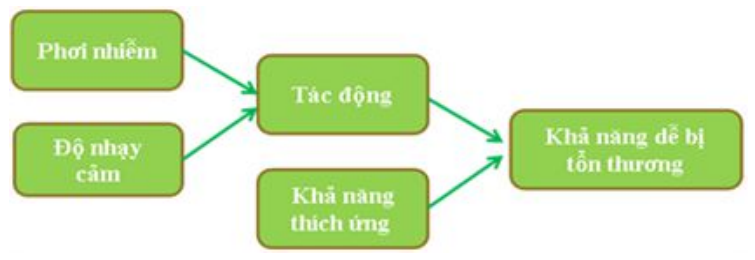

Hình 1. Các thành phần tính dễ bị tổn thương (Fellmann, 2012).
Adger (1999) đã đánh giá tính tổn thương do BĐKH, đặc biệt là các hiện tượng cực đoan khí hậu cho khu vực huyện Xuân Thuỷ, tỉnh Nam Định. Nghiên cứu này đã tập trung vào đánh giá tính tổn thương về khía cạnh xã hội, cụ thể là các tác động bất lợi từ sự thay đổi khí hậu đến sinh kế người dân khu vực nghiên cứu. Trong nghiên cứu này, một số chỉ thị chủ yếu được dùng để đánh giá tính tổn thương như tình trạng nghèo đói, mức độ lệ thuộc vào tài nguyên, khả năng phục hồi, tính bất bình đẳng trong xã hội, mức độ đa dạng đối với nguồn thu nhập của người dân và một số chỉ thị khác liên quan đến các vấn đề về thể chế, chính sách.

Trong dự án xây dựng chỉ số khả năng thích ứng đối với biến đổi khí hậu trong nông nghiệp vùng Prairie của Canada (Swanson, 2007), tác giả chọn lựa được hai mươi chỉ số được tổng hợp từ 6 yếu tố chính: nguồn kinh tế, công nghệ, thông tin, kỹ năng và quản lý, cơ sở hạ tầng và thể chế hóa. Tuy nhiên, tác giả lại xem xét các biến số chỉ số năng lực thích ứng có trọng số ngang nhau trong quá trình tính toán.

Các đánh giá TDBTT dựa trên chỉ số thường xây dựng chỉ số khả năng thích ứng từ kết quả điều tra xã hội học, với các tiêu chí lựa chọn phản ánh được yếu tố tác động và đối tượng bị tác động (thường tập trung vào cộng đồng, xã hội) (Downing, 2001).

Do đó, nhằm đánh giá, phân vùng khả năng thích ứng của vùng nông nghiệp thành phố, nghiên cứu tập trung 2 hướng từ sự hỗ trợ của chính quyền và khả năng tự ứng phó của người dân với các tiêu chí cụ thể về nhận thức về $\mathrm{BĐKH}$, kinh nghiệm ứng phó với BĐKH, sự hỗ trợ của chính quyền và khả năng tiếp cận các nguồn hỗ trợ. Kết quả của nghiên cứu kỳ vọng sẽ là nguồn thông tin hữu ích giúp các ban ngành liên quan, chẳng hạn như Sở Tài ngyên và Môi trường TP.HCM, trong việc ban hành chính sách thích ứng với BĐKH tại TP.HCM.

\section{Vật Liệu và Phương Pháp Nghiên Cứu}

\subsection{Phương pháp AHP}

Khả năng thích ứng của các quận/huyện có hoạt động nông nghiệp được xác định dựa trên các tiêu chí nhận thức về $\mathrm{BDKH}$, kinh nghiệm ứng phó với $\mathrm{BĐKH}$, sự hỗ trợ của chính quyền và khả năng tiếp cận các nguồn hỗ trợ khác. Phương pháp AHP và tham vấn chuyên gia (Saaty, 1988) 
được sử dụng nhằm tính toán trọng số cho các chỉ số đánh giá do BĐKH. Trên cơ sở mục tiêu đánh giá vùng tổn thương do $\mathrm{B} Đ K H$ và các yếu tố ảnh hưởng đến phân vùng tổn thương do $\mathrm{BĐKH} \mathrm{đã}$ được xây dựng, nghiên cứu tiến hành tham vấn 10 chuyên gia đến từ các trường đại học, viện nghiên cứu, cơ quan chính phủ và chính quyền địa phương đại diện cho các lĩnh vực môi trường, BĐKH, nông nghiệp, kinh tế thông qua bảng câu hỏi soạn sẵn.

\subsection{Phương pháp điều tra bằng bảng hỏi}

Khả năng thích ứng của các quận/huyện có hoạt động nông nghiệp được xác định dựa trên kết quả khảo sát cộng đồng và cơ quan quản lý trong lĩnh vực $\mathrm{BĐKH}$, tài nguyên môi trường và nông nghiệp tại thành phố. Để đáp ứng yêu cầu phân tích thống kê cho các nội dung nghiên cứu, bảng câu hỏi soạn sẵn được sử dụng trong quá trình điều tra, với phương thức thực hiện phương pháp điều tra được trình bày khái quát trong Hình 2.

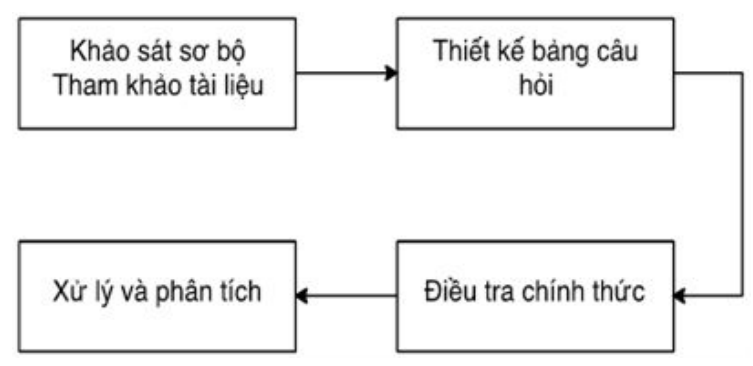

Hình 2. Tiến trình điều tra bằng câu hỏi soạn sẵn.

Về đối tượng và nội dung, quá trình điều tra tập trung vào nhận thức của cán bộ, cơ quan quản lý về $\mathrm{BĐKH} \mathrm{và} \mathrm{năng} \mathrm{lực} \mathrm{thích} \mathrm{ứng,} \mathrm{bao} \mathrm{gồm} \mathrm{toàn} \mathrm{bộ}$ các cơ quan quản lý cấp xã/phường, quận/huyện của TP.HCM (quận/huyện, phường/xã, Sở ngành tham gia Ban chỉ đạo thực hiện kế hoạch hành động ứng phó $\mathrm{BĐKH})$. Bên cạnh đối tượng quản lý, nghiên cứu cũng đã thu thập nhận thức về BĐKH và năng lực thích ứng của động đồng có hoạt động nông nghiệp.

Số lượng phiếu khảo sát được tính với $\mathrm{e}= \pm$ $4 \%$ (Yamane,1967): $\mathrm{n}=\frac{\mathrm{N}}{1+\mathrm{N}\left(\mathrm{e}^{2}\right)}$

Trong đó:

n: số lượng mẫu cần xác định cho nghiên cứu điều tra; $\mathrm{N}$ : tổng số mẫu e: mức độ chính xác mong muốn (1 - độ tin cậy) (chọn độ tin cậy là $96 \%$ )

Theo niên giám thống kê, các quận/huyện có hoạt động nông nghiệp chủ yếu bao gồm Cần Giờ, Nhà Bè, Bình Chánh, Hóc Môn, Củ Chi, Quận 2, 7, 8, 9, 12, Gò Vấp, Tân Bình, Tân Phú, Bình Thạnh, Bình Tân và Thủ Đức, với tổng số hộ nông nghiệp năm 2015 là 24.924 hộ $(\mathrm{N})$. Bên cạnh đó, sai số (e) được chọn là $\pm 4 \%$, nên cỡ mẫu được ước tính là 611 phiếu.

Số phiếu phân bố cho từng quận/huyện được tính toán dựa trên tỷ lệ hộ nông nghiệp và thủy sản trong từng khu vực. Theo đó, số lượng phiếu phân bố cho từng quận/huyện được trình bày trong Bảng 1. Trong đó, các quận 2, 7, 8, Tân Bình, Tân Phú và Bình Thạnh có số phiếu nằm dưới mức đạt ý nghĩa thống kê nên nghiên cứu bỏ qua, tập trung vào đánh giá tổn thương cho các quận/huyện có hoạt động nông nghiệp là chủ yếu.

\subsection{Phương pháp phân tích nhân tố}

Điểm số của từng chỉ số được tính toán bằng cách kết hợp thang đo cảm nhận Likert, phương pháp phân tích nhân tố và phương pháp thống kê (Hair \& ctv., 2006).

\subsubsection{Thang đo cảm nhận Likert}

Nghiên cứu sử dụng thang đo cảm nhận Likert với 5 mức độ để đo lường nhận thức và cảm nhận, với mức độ 1 (biết rất nhiều hoặc hoàn toàn đồng ý) đến 5 (biết rất ít hoặc hoàn toàn không đồng ý) cho 4 tiêu chí. Bảng 2 trình bày chi tiết các biến quan sát cho mỗi tiêu chí tương ứng.

\subsubsection{Phương pháp phân tích nhân tố}

Phương pháp phân tích nhân tố khẳng định (Confirmatory Factor Analysis - CFA) được sử dụng để kiểm định mức độ đại diện cho các biến quan sát của từng tiêu chí. KMO (Kaiser-MeyerOlkin ) là một chỉ tiêu dùng để xem xét sự thích hợp của CFA khi các biến có tương quan với nhau, $0,5 \leq \mathrm{KMO} \leq 1$, phân tích nhân tố được đánh giá thích hợp. Tiêu chuẩn hệ số truyền tải nhân tố (Factor Loading) biểu thị tương quan đơn giữa các biến nhân tố. Theo Hair \& ctv. (2006), Factor Loadings $>0,3$ được xem là đạt mức tối thiểu, Factor Loading $>0,4$ được xem là quan trọng, Factor Loading > 0,5 được xem là có ý nghĩa thực tiễn. Nghiên cứu sử dung phần mềm SPSS 
Bảng 1. Số phiếu phân bố cho từng quận/huyện

\begin{tabular}{|c|c|c|c|c|c|c|}
\hline Quận/huyện & $\begin{array}{c}\text { Hộ trồng } \\
\text { trọt }\end{array}$ & Hộ thủy sản & $\begin{array}{l}\text { Tổng số } \\
\text { hộ trong } \\
\text { Quận/huyện }\end{array}$ & Tỷ lệ (\%) & Số phiếu & $\begin{array}{c}\text { Số phiếu } \\
\text { điều chỉnh } \\
\text { đề phân tích } \\
\text { thống kê }\end{array}$ \\
\hline Cần Giờ & 335 & 3.583 & 3.918 & 15,72 & 98 & 90 \\
\hline Nhà Bè & 96 & 289 & 385 & 1,54 & 10 & 40 \\
\hline Bình Chánh & 3.289 & 392 & 3.681 & 14,77 & 92 & 86 \\
\hline Hóc Môn & 2.538 & 10 & 2.548 & 10,22 & 64 & 64 \\
\hline Củ Chi & 12.236 & 31 & 12.267 & 49,22 & 308 & 131 \\
\hline Quận 2 & 19 & 3 & 22 & 0,09 & 1 & 0 \\
\hline Quận 7 & 4 & 5 & 9 & 0,04 & 0 & 0 \\
\hline Quận 8 & 0 & 13 & 13 & 0,05 & 0 & 0 \\
\hline Quận 9 & 406 & 33 & 439 & 1,76 & 11 & 40 \\
\hline Quận 12 & 945 & 11 & 956 & 3,84 & 24 & 40 \\
\hline Gò Vấp & 118 & 6 & 124 & 0,50 & 3 & 40 \\
\hline Tân Bình & 0 & 0 & 0 & 0,00 & 0 & 0 \\
\hline Tân Phú & 2 & 0 & 2 & 0,01 & 0 & 0 \\
\hline Bình Thạnh & 47 & 2 & 49 & 0,20 & 1 & 0 \\
\hline Bình Tân & 122 & 23 & 145 & 0,58 & 4 & 40 \\
\hline Thủ Đức & 339 & 27 & 366 & 1,47 & 9 & 40 \\
\hline Tổng cộng & & & 24.924 & 100 & & 611 \\
\hline
\end{tabular}

Bảng 2. Các biến quan sát thuộc các tiêu chí đo lường

\begin{tabular}{|c|c|}
\hline Tiêu chí & Biến quan sát \\
\hline $\begin{array}{c}\text { Nhận thức về } \\
\text { BĐKH }\end{array}$ & $\begin{array}{l}\text { Sự hiểu biết của cá nhân đối với vấn đề biến đổi khí hậu }(\mathrm{BDKH}) \\
\text { Sự hiểu biết của cá nhân về khái niệm, nguyên nhân, và cách giải quyết } \\
\text { toàn cầu đối với BĐKH } \\
\text { Sự quan tâm của cá nhân đối với vấn đề BĐKH } \\
\text { Ânh hưởng của các biểu hiện BĐKH như nhiệt độ tăng, mưa bão, xâm nhập } \\
\text { mặn đến đời sống cá nhân và gia đình } \\
\text { Sự cảm nhận về thay đổi thời tiết trong vòng } 5 \text { năm qua }\end{array}$ \\
\hline $\begin{array}{l}\text { Kinh nghiệm thích } \\
\text { ứng từ người dân }\end{array}$ & $\begin{array}{l}\text { Phương pháp thích ứng với } \mathrm{BĐKH} \mathrm{như} \mathrm{trồng} \mathrm{cây} \\
\text { Phương pháp thích ứng với } \mathrm{BĐKH} \mathrm{như} \mathrm{cài} \mathrm{đặt} \mathrm{máy} \mathrm{điều} \mathrm{hòa} \mathrm{nhiệt} \mathrm{độ} \\
\text { Phương pháp thích ứng với } \mathrm{BĐKH} \mathrm{như} \mathrm{thay} \mathrm{đổi} \mathrm{cấu} \mathrm{trúc} \mathrm{nhà} \mathrm{ở} \\
\text { Phương pháp thích ứng với } \mathrm{BĐKH} \mathrm{như} \mathrm{di} \mathrm{chuyển} \mathrm{đến} \mathrm{nơi} \mathrm{khác}\end{array}$ \\
\hline $\begin{array}{c}\text { Khả năng tiếp cận } \\
\text { thông tin BĐKH }\end{array}$ & $\begin{array}{l}\text { Phương pháp chuyển đổi giống cây trồng, vật nuôi } \\
\text { Thông tin về quy hoạch thủy lợi, chống ngập úng liên quan đến BĐKH } \\
\text { Thông tin về nâng cao chất lượng nguồn nhân lực và tuyên truyền, nâng } \\
\text { cao nhận thức cộng đồng trong xây dựng năng lực chủ động ứng phó với } \\
\text { BĐKH của TP.HCM } \\
\text { Thông tin về cải tạo khu công viên, cây xanh hiện hữu trong đô thị; phát } \\
\text { triển bảo vệ khu dự trữ sinh quyền rừng ngập mặn Cần Giờ } \\
\text { Thông tin về lồng ghép các yếu tố BĐKH vào các Chiến lược, Chương trình, } \\
\text { Quy hoạch và Kế hoạch phát triển kinh tế - xã hội của TP.HCM } 2017 \text { - } 2020\end{array}$ \\
\hline $\begin{array}{l}\text { Mức độ hỗ trợ } \\
\text { từ phía chính quyền } \\
\text { đến } \mathrm{BĐKH}\end{array}$ & $\begin{array}{l}\text { Sự cần thiết của các hoạt động tuyên truyền } \mathrm{BDKH} \\
\text { Sự nỗ lực giải quyết vấn đề BĐKH tại TP.HCM } \\
\text { Sự nỗ lực đưa ra các chương trình thích nghi với BĐKH tại TP.HCM } \\
\text { Sự hiệu quả của các biện pháp và chương trình thích nghi với BĐKH }\end{array}$ \\
\hline
\end{tabular}


20 cho phân tích nhân tố.

\subsubsection{Phương pháp thống kê}

Sau khi có kết quả từ phân tích nhân tố, chỉ số thống kê là trung bình điểm số (Average Score) của những biến quan sát tương quan với nhau để đo lường 4 tiêu chí, với công thức tính điểm trung bình cho một tiêu chí được đưa ra như sau:

Điểm trung bình = Tổng điểm của các biến quan sát có tương quan / Tổng số mẫu quan sát

\subsection{Phương pháp chuẩn hoá số liệu cho các tiêu chí đánh giá khả năng thích ứng}

Trong nghiên cứu này, các giá trị của từng yếu tố khả năng thích ứng đều được chuẩn hoá theo Hướng dẫn đánh giá xã hội học xác định chỉ số phát triển con người (HDI) từ UNDP (2006).

$$
\begin{aligned}
\mathrm{x}_{\mathrm{ij}} & =\frac{\mathrm{X}_{\mathrm{ij}}-\operatorname{Min}_{\mathrm{i}}\left\{\mathrm{X}_{\mathrm{ij}}\right\}}{\operatorname{Max}_{\mathrm{i}}\left\{\mathrm{X}_{\mathrm{ij}}\right\}-\operatorname{Min}_{\mathrm{i}}\left\{\mathrm{X}_{\mathrm{ij}}\right\}} \\
\mathrm{x}_{\mathrm{ij}} & =\frac{\operatorname{Max}_{\mathrm{i}}\left\{\mathrm{X}_{\mathrm{ij}}\right\}-\mathrm{X}_{\mathrm{ij}}}{\operatorname{Max}_{\mathrm{i}}\left\{\mathrm{X}_{\mathrm{ij}}\right\}-\operatorname{Min}_{\mathrm{i}}\left\{\mathrm{X}_{\mathrm{ij}}\right\}}
\end{aligned}
$$

Trong đó: $\mathrm{x}_{\mathrm{ij}}$ : giá trị sau khi chuẩn hoá $(0 \leq$ $\left.\mathrm{x}_{\mathrm{ij}} \leq 1\right)$, Xij: giá trị thực

i: các chỉ thị đánh giá $(\mathrm{i}=1, \ldots, 4)$, j: các quận/huyện đánh giá $(\mathrm{j}=1, \ldots, 10)$

Các giá trị khả năng thích ứng được phân thành 5 cấp (Nguyen, 2006) như sau:

Khả năng thích ứng rất thấp: $0,00 \leq \mathrm{x}_{\mathrm{ij}} \leq 0,20$

Khả năng thích ứng thấp: $0,21 \leq \mathrm{x}_{\mathrm{ij}} \leq 0,40$ 0,60

Khả năng thích ứng trung bình: $0,41 \leq \mathrm{x}_{\mathrm{ij}} \leq$

Khả năng thích ứng cao: $0,61 \leq \mathrm{x}_{\mathrm{ij}} \leq 0,80$

Khả năng thích ứng rất cao: $0,81 \leq \mathrm{x}_{\mathrm{ij}} \leq 1,00$

\section{Kết Quả và Thảo Luận}

Trong khuôn khổ nghiên cứu này, tính tổn thương do $\mathrm{BĐKH}$ được xác định chi tiết đến cấp quận, cụ thể là tập trung vào 10 quận/huyện có hoạt động nông nghiệp (trồng trọt và nuôi trồng thủy sản) chủ yếu trên địa bàn TP.HCM (Hình 3), bao gồm các quận/huyện Bình Chánh, Bình Tân, Cần Giờ, Củ Chi, Gò Vấp, Hóc Môn, Nhà Bè, Quận 12, Quận 9 và thủ Đức. Bảng 3 trình bày đặc điểm kinh tế - xã hội của người được khảo sát và hộ với số lượng nam giới chiếm $57 \%$, độ tuổi từ 30 đến 49 tuổi chiếm $54 \%$, và phần lớn có trình độ đại học chiếm 67\% trong tổng số hộ khảo sát.

Bảng 3. Đặc điểm kinh tế - xã hội của mẫu khảo

\begin{tabular}{|c|c|}
\hline Các yếu tố & Tỷ lệ (\%) \\
\hline \multicolumn{2}{|c|}{ Đặc điểm của người được phỏng vấn } \\
\hline \multicolumn{2}{|l|}{ Giới tính } \\
\hline Nam & 56,86 \\
\hline Nữ & 43,14 \\
\hline \multicolumn{2}{|l|}{ Tuổi (năm) } \\
\hline$\leq 29$ & 32,27 \\
\hline $30-49$ & 53,68 \\
\hline$>50$ & 14,05 \\
\hline \multicolumn{2}{|l|}{ Trình độ học vấn } \\
\hline Cấp 1 & 10,20 \\
\hline Cấp 2 & 8,00 \\
\hline Cấp 3 & 15,10 \\
\hline Đại học & 66,70 \\
\hline \multicolumn{2}{|l|}{ Nghề nghiệp } \\
\hline Nội trợ & 4,18 \\
\hline Sinh viên & 11,04 \\
\hline Công nhân & 9,04 \\
\hline Khác & 75,75 \\
\hline \multicolumn{2}{|l|}{ Đặc điểm của hộ } \\
\hline \multicolumn{2}{|l|}{ Quy mô hộ (người) } \\
\hline$\leq 5$ & 82,94 \\
\hline$>5$ & 17,06 \\
\hline \multicolumn{2}{|l|}{ Tình trạng cư trú } \\
\hline Tạm trú dài hạn & 88,47 \\
\hline Tạm trú ngắn hạn & 11,53 \\
\hline \multicolumn{2}{|c|}{ Thu nhập (triệu VND/tháng) } \\
\hline$\leq 10$ & 70,9 \\
\hline$\overline{10}-12$ & 28,93 \\
\hline$>20$ & 0,17 \\
\hline
\end{tabular}
sát

Các phiếu tham vấn chuyên gia được thực hiện nhằm tính toán trọng số cho từng tiêu chí, chi tiết trình bày trong Hình 4 .

Sau khi phân tích CFA cho 4 tiêu chí gồm (i) nhận thức của người dân về $\mathrm{BĐKH,} \mathrm{(ii)} \mathrm{kinh}$ nghiệm ứng phó với $\mathrm{BĐKH}$ từ người dân, (iii) khả năng tiếp cận thông tin về $\mathrm{BĐKH} \mathrm{do} \mathrm{người}$ dân đánh giá, (iv) sự hỗ trợ từ chính quyền do người dân và công chức viên chức đánh giá và lần lượt loại bỏ các biến không đạt yêu cầu (hệ số tải nhỏ hơn 0,3 ). Nghiên cứu tính điểm trung bình cho mỗi tiêu chí theo công thức (2) và thu được kết quả như trình bày trong Bảng 3 . Theo đó, tất cả 4 tiêu chí điều thể hiện mối quan hệ thuận (+) với yếu tố khả năng thích ứng. Kết quả cho 


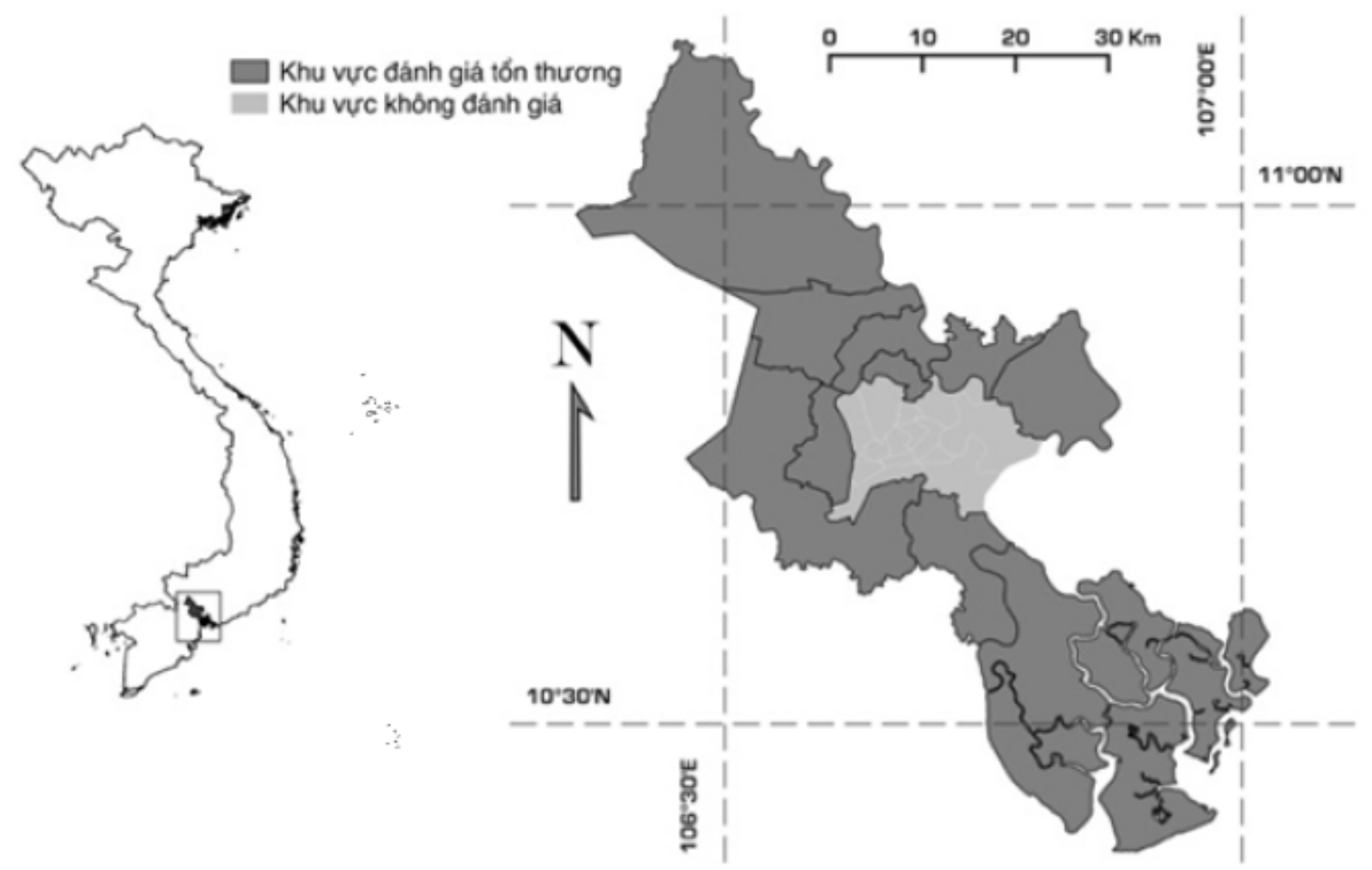

Hình 3. Khu vực các quận/huyện đánh giá tính tổn thương.

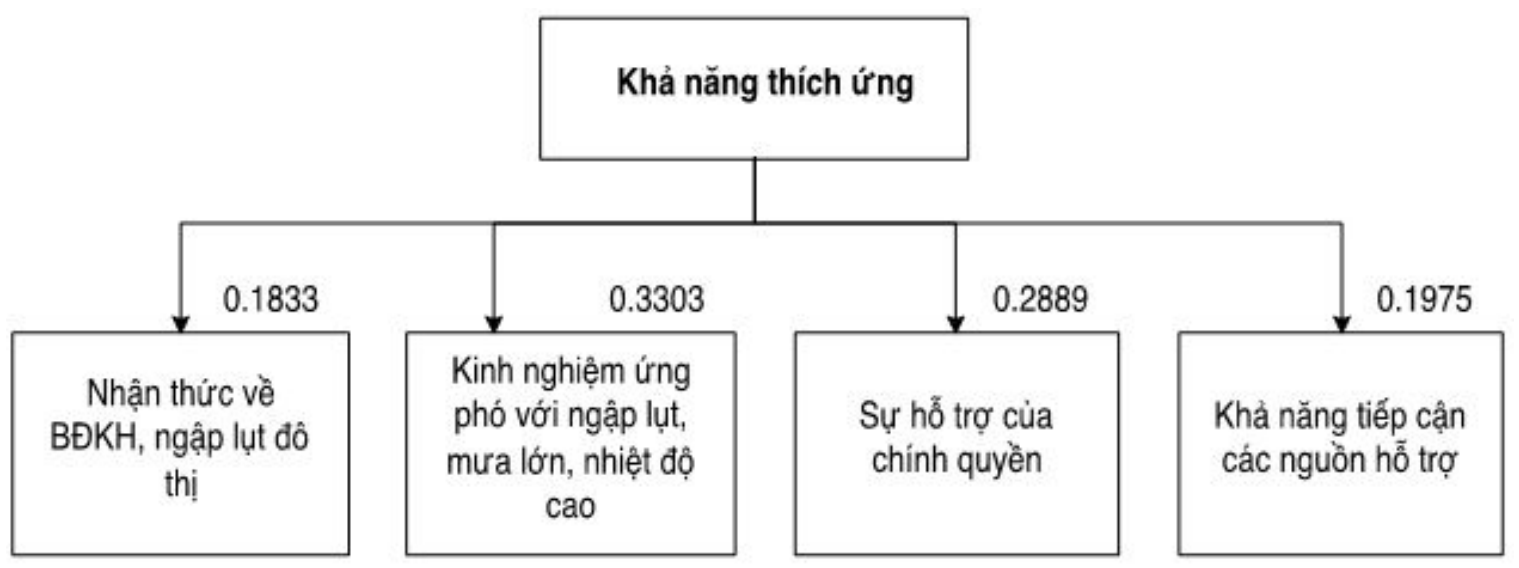

Hình 4. Khu vực các quận/huyện đánh giá tính tổn thương.

thấy nhận thức của người dân về $\mathrm{BĐKH} \mathrm{ở} \mathrm{các}$ quận/huyện là khá tốt (3,00 - 3,92 điểm), kinh nghiệm ứng phó với $\mathrm{BĐKH}$ của người dân các quận khá cao (3,00 - 3,85 điểm), khả năng tiếp cận thông tin về BĐKH còn chưa nhiều ở quận Gò Vấp (2,44 điểm) và khá nhiều ở quận Củ Chi $(3,66$ điểm), và hỗ trợ chính quyền do người dân đánh giá là chưa cao ở quận 12 (2,61 điểm) và khá cao ở quận Củ Chi (4,02 điểm). Theo công chức viên chức đánh giá, sự hỗ trợ của chính quyền đối với vấn đề $\mathrm{BĐKH} \mathrm{là} \mathrm{khá} \mathrm{tốt} \mathrm{với} \mathrm{3,40} \mathrm{điểm.} \mathrm{Để} \mathrm{thích}$ nghi hơn với $\mathrm{BDKH}$, người dân TP.HCM đa số trồng thêm cây xanh tạo bóng mát và không gian xanh cho gia đình (chiếm $42 \%$ tổng số hộ khảo sát), lắp đặt máy điều hòa để làm mát (chiếm $25 \%$ tổng số hộ khảo sát), thay đổi cấu trúc nhà 
Bảng 4. Giá trị các tiêu chí cho đánh giá khả năng thích ứng

\begin{tabular}{lcccc}
\hline & $\begin{array}{c}\text { Nhận thức về } \\
\text { BĐKH, ngập } \\
\text { lụt đô thị }\end{array}$ & $\begin{array}{c}\text { Kinh nghiệm ứng phó } \\
\text { với ngập lụt, mưa lớn, } \\
\text { nhiệt độ cao }\end{array}$ & $\begin{array}{c}\text { Sự hỗ trợ của } \\
\text { chính quyền }\end{array}$ & $\begin{array}{c}\text { Khả năng tiếp } \\
\text { cận các nguồn } \\
\text { hỗ trợ }\end{array}$ \\
\hline (Mối quan hệ) & $(+)$ & $(+)$ & $(+)$ & $(+)$ \\
Bình Chánh & 3,36 & 3,01 & 2,89 & 2,89 \\
Bình Tân & 3,43 & 3,33 & 2,79 & 2,78 \\
Cần Giờ & 3,58 & 3,22 & 3,08 & 2,73 \\
Củ Chi & 3,55 & 3,68 & 4,02 & 3,66 \\
Gò Vâ̂p & 3,17 & 3,12 & 2,96 & 2,44 \\
Hóc Môn & 3,54 & 3,51 & 2,61 & 2,79 \\
Nhà Bè & 3,92 & 3,10 & 2,73 & 2,75 \\
Quận 12 & 3,38 & 3,51 & 3,22 & 2,81 \\
Quận 9 & 3,11 & 3,85 & 3,24 & 2,51 \\
Thủ Đức & 3,00 & 3,00 & 3,10 & 2,94 \\
\hline
\end{tabular}

${ }^{1} \mathrm{BĐKH}$ : biến đổi khí hậu.
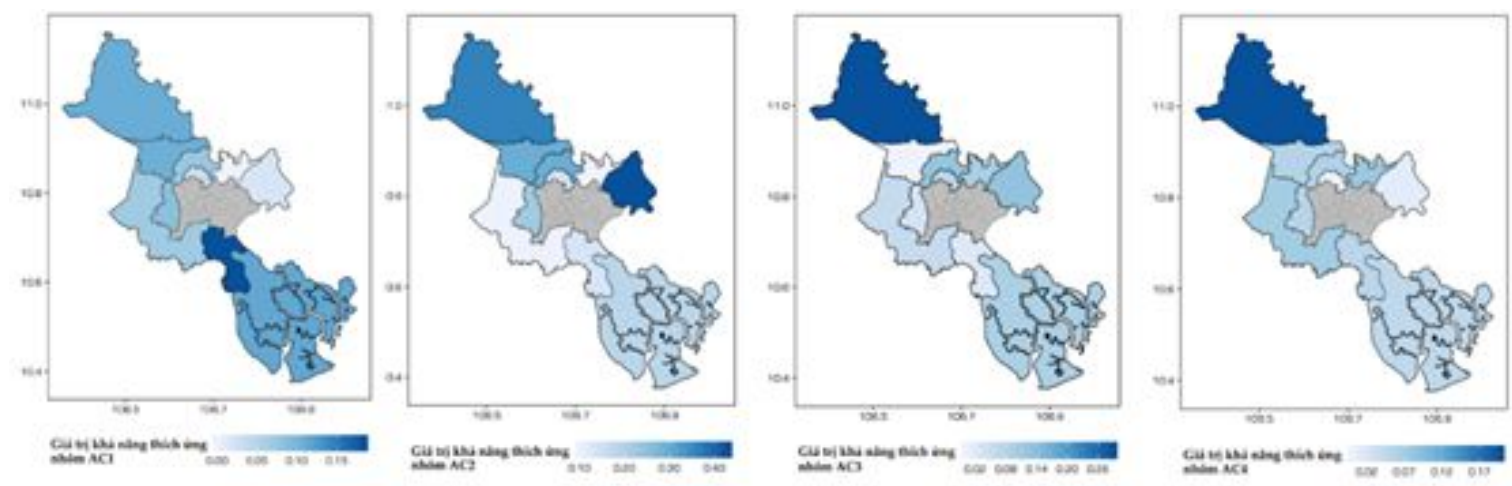

Hình 5. Phân vùng các giá trị của từng tiêu chí đánh giá khả năng thích ứng với BĐKH.

ở để thích ứng với sự tăng nhiệt độ (chiếm $16 \%$ tổng số hộ khảo sát), chuyển đổi giống cây trồng vật nuôi (chiếm 14\% tổng số hộ khảo sát), ngoài ra một số rất ít hộ lựa chọn di chuyển đến nơi khác (chỉ chiếm $3 \%$ tổng số hộ khảo sát).

Dựa trên kết quả thu thập và xử lý số liệu thôn cho 4 tiêu chí đánh giá khả năng thích ứng, nghiên cứu tiến hành chuẩn hóa giá trị cho các tiêu chí và xác định giá trị khả năng thích ứng tại mỗi quận/huyện từ các giá trị chuẩn hóa và giá trị trọng số. Theo đó, kết quả xác định giá trị khả năng thích ứng tổng hợp cho các quận/huyện được trình bày trong Bảng 4 .

Nghiên cứu tiến hành xây dựng bản đồ phân bố khả năng thích ứng dựa trên kết quả tính toán giá trị khả năng thích ứng tổng hợp cho từng quận/huyện (Hình 5). Bên cạnh đó, Hình 6 cũng lần lượt mô tả lại về mặt không gian cho các giá trị khả năng thích ứng tương ứng với mỗi tiêu chí đánh giá trong nghiên cứu này.

Kết quả tính toán giá trị và thành lập bản đồ cho thấy huyện Củ Chi được đánh giá có khả năng thích ứng cao nhất, với giá trị khả năng thích ứng tổng thể là 0,86 . Theo đó, huyện $\mathrm{Củ}$ Chi được ghi nhận đồng thời có sự hỗ trợ từ chính quyền và khả năng tiếp cận các nguồn hỗ trợ cao nhất trong số 10 quận/huyện. Hơn nữa, huyện Củ Chi cũng được xếp hạng cao khi xét riêng hai tiêu chí đánh giá khả năng thích ứng còn lại, cụ thể là lần lượt xếp thứ 2 và thứ 3 đối với tiêu chí kinh nghiệm ứng phó với các hiện tượng cực đoan và nhận thức về BĐKH, ngập lụt đô thị (Bảng 5).

Tương tự, kết quả phân tích cho thấy quận 9 có khả năng thích ứng cao hơn các quận/huyện còn lại. Theo đó, quận 9 là khu vực có kinh nghiệm ứng phó với các hiện tượng cực đoan khí hậu cao nhất và cũng đồng thời là khu vực có giá trị cao thứ hai khi xét đến tiêu chí sự hỗ trợ từ chính 


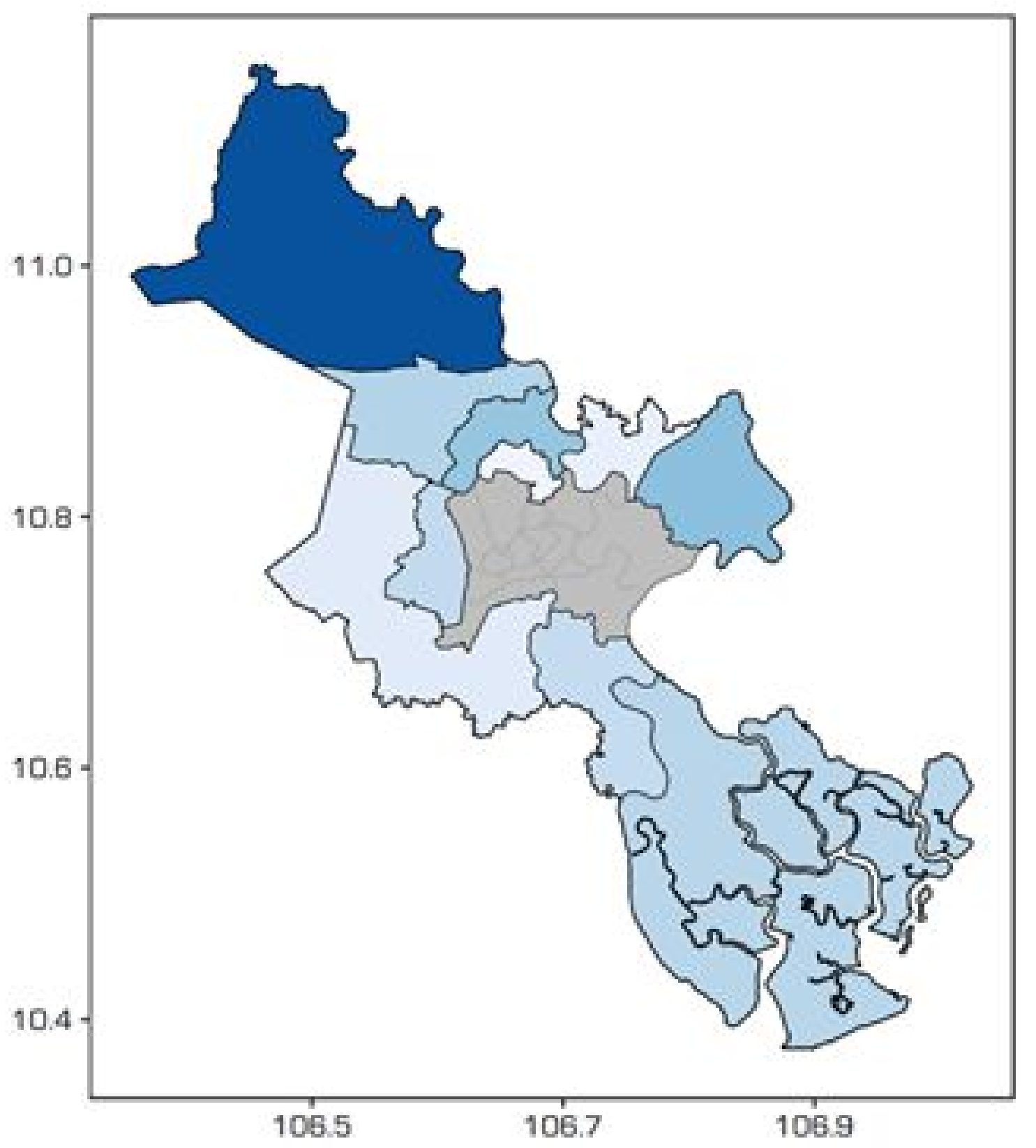

\section{Giá trị khả năng thich ứng}

Hình 6. Bản đồ phân bố giá trị khả năng thích ứng tổng thể.

quyền. Đây là hai tiêu chí này được các chuyên gia nhận định có ý nghĩa quan trọng nhất trong số các tiêu chí được lựa chọn trong quá trình đánh giá khả năng thích ứng.

Bên cạnh đó, quận 12 được xếp hạng thứ 3 về khả năng thích ứng, cũng được ghi nhận có giá 
Bảng 5. Giá trị khả năng thích ứng tổng hợp cho các quận/huyện

\begin{tabular}{|c|c|c|c|c|c|}
\hline & $\begin{array}{c}\text { Nhận thức về } \\
\text { BĐKH, ngập } \\
\text { lụt đô thị }{ }^{1}\end{array}$ & $\begin{array}{l}\text { Kinh nghiệm } \\
\text { ứng phó với } \\
\text { ngập lụt, } \\
\text { mưa lớn, } \\
\text { nhiệt độ cao }\end{array}$ & $\begin{array}{l}\text { Sự hỗ trợ của } \\
\text { chính quyền }\end{array}$ & $\begin{array}{l}\text { Khả năng } \\
\text { tiếp cận các } \\
\text { nguồn hỗ trợ }\end{array}$ & $\begin{array}{l}\text { Giá trị khả } \\
\text { năng thích } \\
\text { ứng (AC) }\end{array}$ \\
\hline (Mối quan hệ) & $\overline{(+)}$ & $(+)$ & $(+)$ & $(+)$ & \\
\hline Bình Chánh & 0,0712 & 0,0039 & 0,0566 & 0,0728 & 0,2045 \\
\hline Bình Tân & 0,0859 & 0,1282 & 0,0363 & 0,0550 & 0,3055 \\
\hline Cần Giờ & 0,1156 & 0,0855 & 0,0963 & 0,0469 & 0,3444 \\
\hline $\mathrm{Củ} \mathrm{Chi}$ & 0,1107 & 0,2642 & 0,2889 & 0,1975 & 0,8613 \\
\hline Gò Vấp & 0,0335 & 0,0466 & 0,0717 & 0,0000 & 0,1519 \\
\hline Hóc Môn & 0,1073 & 0,1982 & 0,0000 & 0,0567 & 0,3621 \\
\hline Nhà Bè & 0,1833 & 0,0389 & 0,0251 & 0,0502 & 0,2974 \\
\hline Quận 12 & 0,0755 & 0,1982 & 0,1250 & 0,0599 & 0,4586 \\
\hline Quận 9 & 0,0208 & 0,3303 & 0,1291 & 0,0113 & 0,4915 \\
\hline Thủ Đức & 0,0000 & 0,0000 & 0,1004 & 0,0809 & 0,1813 \\
\hline
\end{tabular}

${ }^{1} \mathrm{BĐKH:} \mathrm{biến} \mathrm{đổi} \mathrm{khí} \mathrm{hậu.}$

trị khả năng thích ứng tổng thể tương đối cao, cụ thể là 0,459 . Trong khi đó, quận Gò Vấp là khu vực được đánh giá có khả năng thích ứng thấp nhất trong số 10 quận/huyện nghiên cứu, với giá trị tổng hợp là 0,151 .

Với việc sử dụng phương pháp điều tra xã hội học kết hợp giữa nhận thức và kinh nghiệm ứng phó BĐKH của cộng đồng ở các quận/huyện hoạt động nông nghiệp, kinh nghiệm và sự hỗ trợ từ phía chính quyền, nghiên cứu đã phân vùng khả năng thích ứng với BĐKH cho ngành nông nghiệp Thành phố. Trong đó, các quận huyện có khả năng thích ứng cao là Củ Chi. Các quận 9, 12 cũng là những huyện có khả năng thích ứng trung bình. Trong khi đó, Bình Chánh, Gò Vấp, Thủ Đức nằm trong khu vực có khả năng thích ứng thấp.

\section{Kết Luận}

Qua kết quả nghiên cứu, khả năng thích ứng của 10 quận/huyện được xác định dựa vào 4 tiêu chí, cự thể là (i) nhận thức về $B Đ K H$ và ngập lụt đô thị, (ii) kinh nghiệm ứng phó với ngập lụt, mưa lớn và nhiệt độ cao, (iii) sự hỗ trợ của chính quyền và (iv) khả năng tiếp nhận các nguồn hỗ trợ dựa trên các giá trị tổng hợp từ quá trình điều tra, khảo sát tại khu vực nghiên cứu và xếp hạng được mức độ dựa trọng số riêng cho mỗi tiêu chí. Nhìn chung, kết quả nghiên cứu này đã cho thấy được khả năng thích ứng của khu vực nghiên cứu, làm nền tảng cho bước xác định tính tổn thương cũng như cho việc thành lập bản đồ tổn thương trước tác động BĐKH.

Về khía cạnh phương pháp, hướng tiếp cận trong nghiên cứu này cơ bản đã xác định mức độ khả năng thích ứng và xây dựng thành công bản đồ khả năng thích ứng cho mỗi quận/huyện. Tuy nhiên, khả năng thích ứng nói riêng cũng như tính tổn thương nói chung của một khu vực nhất được là do đóng góp từ nhiều yếu tố hay (nhóm) tiêu chí. Hơn nữa, mỗi (nhóm) tiêu chí cũng có mức độ ưu tiên khác nhau (được gán trọng số khác nhau), vì vậy rất khó xác định được tỷ lệ đóng góp hay nguyên nhân chính dẫn đến khả năng thích ứng cao/thấp tại một khu vực nhất định. Do đó, hướng nghiên cứu tiếp theo có thể làm rõ hơn khía cạnh này dựa trên ứng dụng một số phương pháp thống kê như phân tích thành phần chính (PCA) hay những phương pháp tương tự đã được áp dụng thành công trước đây.

\section{Lời Cảm Ơn}

Nghiên cứu này là một phần kết quả Đề tài Ứng dụng GIS và mô hình hóa cho bản đồ đánh giá tính dễ bị tổn thương của BĐKH ở Thành phố Hồ Chí Minh và đề xuất giải pháp thích ứng giai đoạn đến năm 2050 theo hợp đồng số 29/2017/HĐSKHCN ngày 31/10/2017 với Viện Khoa học và Công nghệ Tính Toán Thành phố Hồ Chí Minh. Nhóm nghiên cứu trân trọng cám ơn Sở Khoa học và Công nghệ và Viện Khoa học và Công nghệ Tính Toán Thành phố Hồ Chí Minh cấp kinh phí 
cho Đề tài này. Ngoài ra, các tác giả cũng chân thành cảm ơn ThS. Đặng Nguyễn Đông Phương đã hỗ trợ kỹ thuật xây dựng bản đồ cho nghiên cứu này.

\section{Tài Liệu Tham Khảo (References)}

ADB (Asean Development Bank). (2013). Vietnam: Environment and climate change assessment. Mandaluyong, Philippines: ADB.

ADB (Asean Development Bank). (2010). Ho Chi Minh City adaptation to climate change: summary report. Mandaluyong, Philippines: ADB.

Adger W. N. (1999). Social vulnerability to climate change and extremes in coastal Vietnam. World Development 27(2), 249-269.

Balica, S. F., Wright, N. G., \& van der Meulen, F. (2012). A flood vulnerability index for coastal cities and its use in assessing climate change impacts. Natural Hazards 64(1), 73-105.

Can, V. T., Nguyen, S. T., Tran, A. N., \& Dang, K. D. (2013). Vulnerability assessment methods - Theory and practice. Part 2. Applying the calculation test for vulnerable indexes due to flood in Lam river basin - Nghe An province. VNU Journal of Science: Natural Sciences and Technology 29(2S), 223-232.

DONRE (Department of Natural Resources and Environment). (2010). Climate change adaptation and mitigation action plan report. Ho Chi Minh City, Vietnam: DONRE Office.

Downing, T. E., Butterfield, R., Cohen, S., Huq, S., Moss, R., Rahman, A., Sokona, Y., \& Stephen, L. (2001). UNEP vulnerability indices: Climate change impacts and adaptation. UNEP policy series. Nairobi, Kenya: UNEP.

Fellmann, T. (2012). The assessment of climate changerelated vulnerability in the agricultural sector: reviewing conceptual frameworks. In Meybeck, S., Lankoski, J., Redfern, S., Azzu, N., \& Gitz, V. (Eds.) Building resilience for adaptation to climate change in the agriculture sector (37-61). Rome, Italia: FAO and OECD.

GSO (General Statistical Office). (2016). Vietnam population forecast 2014 - 2049. Ha Noi, Vietnam: Statistical Publisher.
Hair, J. F., Black, B., Babin, B., Anderson, R. E., \& Tatham, R. L. (2006). Multivariate data analysis $\left(6^{\text {th }}\right.$ ed.). London, UK: Pearson Prentice Hall.

IPCC (The Intergovernmental Panel on Climate Change). (2014). Climate change 2014: impacts, adaptation, and vulnerability. Part A: Global and sectoral aspects. Contribution of working group II to the fifth assessment report of the intergovernmental panel on climate change. New York, USA: Cambridge University Press.

Kumar, P., Geneletti, D., \& Nagendra, H. (2016). Spatial assessment of climate change vulnerability at city scale: A study in Bangalore, India. Land Use Policy $58,514-532$.

MONRE (Ministry of Natural Resources and Environment). (2016). Climate change and sea level rise scenarios for Viet Nam. Ha Noi, Vietnam: Vietnam Natural Resources and Environment Publishing House.

Saaty, T. L. (1988). What is the analytic hierarchy process? In Mitra, G. (Ed.). Mathematical models for decision support (109-121). Berlin, Germany: Springer.

Swanson, D., Hiley, J., Venema, H., \& Grosshams, R. (2007). Indicators of adaptive capacity to climate change for agriculture in the Prairie region of Canada: An analysis based on statistics Canada's census of agriculture. Working Paper for the Prairie Climate Resilience Project. Winnipeg, Manitoba: International Institute for Sustainable Development.

Tri, V. P. D., Trung, N. H., \& Thanh, V. Q. (2013). Vulnerability to flood in the Vietnamese Mekong delta: Mapping and uncertainty assessment. Journal of Environmental Science and Engineering B(2), 514-532.

UNDP (United Nations Development Programme). (2006). Human development report 2006 beyond scarcity: Power, poverty and the global water crisis. New York, USA: Palgrave Macmillan.

Yamane, T. (1967). Statistics: An introductory analysis ( $2^{\text {nd }}$ ed.). New York, USA: Harper \& Row. 